Research Article

\title{
Insights into the Binding of 3-(I-Phenylsulfonyl- 2-methylindol-3-ylcarbonyl) Propanoic Acid to Bovine Serum Albumin: Spectroscopy and Molecular Modelling Studies
}

\author{
Subramani Karthikeyan ${ }^{1}$, Shanmugavel Chinnathambi ${ }^{1}$, Devadasan Velmurugan ${ }^{2,3}$, \\ Ganesan Bharanidharan ${ }^{1}$, Singaravelu Ganesan ${ }^{1}$ \\ ${ }^{1}$ Department of Medical physics, Anna University, Chennai-600 025, India \\ ${ }^{2}$ Center of Advanced Study in Crystallography and Biophysics, University of Madras, Chennai -600 025, India \\ ${ }^{3}$ Bioinformatics Infrastructure Facility, University of Madras, Chennai-600 025, India
}

Corresponding author: E-mail: sganesan@annauniv.edu, ganesansingaravelu@gmail.com

Received: Nov. 26, 20I4; Accepted: Jan. 5, 20I5; Published: Jan. 10, 2015.

Citation: Subramani Karthikeyan, Shanmugavel Chinnathambi, Devadasan Velmurugan, Ganesan Bharanidharan and Singaravelu Ganesan. Insights into the Binding of 3-(I-Phenylsulfonyl-2-methylindol-3-ylcarbonyl) Propanoic Acid to Bovine Serum Albumin: Spectroscopy and Molecular Modelling Studies. Nano Biomed. Eng. 20I5, 7(I), I-7.

DOI: $10.5101 / \mathrm{nbe.v7il.pl-7.}$

\begin{abstract}
Serum albumin is a globular protein which is most abundant in human that binds remarkably with wide range of drugs. A reliable prediction of protein and drug binding at the atomic level by optical spectroscopy and molecular modeling methods provides the basis for the design of new drug compounds. In the current study, A newly synthesized 3-(1-Phenylsulfonyl-2-methylindol-3ylcarbonyl) propanoic acid (PA) which has a antifungal and anti bacterial effects also plays vital role for the nutrition, micro biome and physiology triangle. It has been reported that $90 \%$ of PA quantity is metabolized by the liver and the rest is transported into the peripheral blood, since PA has binding characteristics, understanding pharmacokinetic mechanism of the drug is important. In this regard, the binding of PA-Bovine Serum Albumin (BSA) was investigated by UV-Vis, fluorescence spectroscopy and molecular docking studies. From the experimental and modeling studies it is observed that PA could bind BSA through the hydrophobic force, and hydrogen bonding. The current study reveals that the optical spectroscopy and molecular modeling techniques could be effectively used to study the design of new drug and understanding their pharmacokinetics.
\end{abstract}

Keywords: Bovine Serum Albumin; Propanoic Acid; Fluorescence Quenching; Fluorescence Resonance Energy Transfer; Molecular docking

\section{Introduction}

A large body of research indicates that dietary fiber has a profound effect on general health, which includes the increase of post-meal satiety, decrease in body weight, fat mass and the severity of diabetes
[1]. These effects may be contributed due to the fermentation of dietary fiber by the colonic microbiota and in turn might be the cause for the production of various metabolites, which are absorbed by the host and influenceits energy homeostasis. The microbiota also influences the development of obesity and its 
associated diseases.

Proponic acid (PA) is an important link in the nutrition, micro biome and physiology triangle and also occurs naturally in food products like milk in low quantity and relatively high in dairy products such as yogurt and cheese as a bacterial fermentation, mostly for propioni bacteria [2]. For a long period, since propanoic acid has been under estimated in terms of its physiological impact; most studies have addressed the effects of butyrate but acetate to a lesser extent. Although the butyrate and acetate probably have importance in intestinal physiology, systemically they have less significant effects. In addition to these properties, PA also has anti-fungal and bacterial effects.

PA is mainly produced in the colon by the fermentation of indigested food made by the microbiota. When it reaches the blood compartment and the adipose tissue, it reduces fatty acid levels in plasma via inhibition of lipolysis; further lipogenesis takes place in adipose tissue and suppress the production of fatty acid in liver. From this, we have understood that this is a major mechanism by which prebiotics exert their effects on obesity-related disease. Lowering the presence of fatty acid in the plasma may be especially important; because it is known that higher amount of fatty acid in plasma can cause inflammation. Moreover, it is also known that fatty acid and inflammatory factors cause insulin resistance [6-10]. Although, around $90 \%$ of PA quantity is metabolized by the liver and the rest is transported into the peripheral blood [4]; in humans its quantity was reported to be in the range of $6 \mu \mathrm{M}[3,4]$. This brings us a huge interest to understand and establish the pharmacokinetics PA with serum albumin.

Serum albumin is the major component in plasma protein for human beings. This globular protein, which consists of a single polypeptide chain of 585 amino acid residues, has important physiological functions involving in the transport and distribution of many molecules and metabolites, such as fatty acids, amino acids, hormones, cations and anions, and many diverse drugs, including anticoagulants, tranquilizers, and anesthetics. It has been shown that the distribution, free concentration, and metabolism of various drugs can be significantly altered because of their binding to BSA $[12,13]$. The only tryptophan (Trp) residue is located in the hydrophobic cavity, and thus the study of its interaction with small molecules can provide useful insights to understanding environment-dependent molecular interactions [14]. Moreover, there is evidence of conformational changes of serum albumin induced by its interaction with low molecular weight drugs, which appear to affect secondary and tertiary structures of proteins $[15,16]$.

Herein, we have discuss the synthesised drug of 3-(1-Phenylsulfonyl-2-methylindol-3-ylcarbonyl) propanoic acid with bovine serum albumin, the protein conformational studies using different optical spectroscopic techniques using UV-Visible, fluorescence emission spectroscopy, the binding mode of PA with complex BSA also studied by molecular docking.

\section{Materials}

Bovine serum albumin was purchased from $\mathrm{Hi}$ Media laboratory Pvt. Limited, Mumbai, India and it was used without any further purification. Millipore water was used for preparing solution throughout the experiments. BSA solution was prepared in Tris $-\mathrm{HCl}$ buffer solution of $\mathrm{pH}$ 7.4. BSA solution was kept in the dark at $4^{\circ} \mathrm{C}$. 3-(1-Phenylsulfonyl-2-methylindol3 -ylcarbonyl) propanoic compound was prepared by the Friedel \pm Crafts acylation of 1-phenylsulfonyl-2methylindole with succinic anhydride in the presence of anhydrous aluminium chloride in dry methylene chloride21 (Fig.1).

\section{Methods}

Absorption spectra were recorded in the wavelength range of 190-400 nm using UV-Visible absorption spectrophotometer (Perkin-Elmer Lamda35, Waltham, MA). The steady state fluorescence emission measurements were obtained using a commercially available spectrofluorometer (Fluoromax-2, ISA;

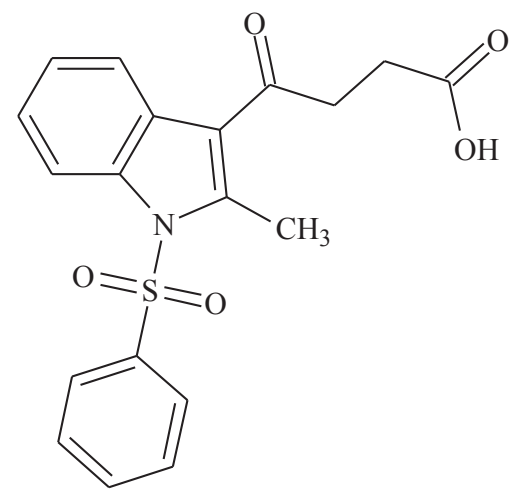

Fig. 1 Chemical structure of propanic acid (PA). 
Jobin-Yuvon-Spex, Edison, NJ) and spectral band passes were kept as $5 \mathrm{~nm}$ in both excitation and emission monochromators. The emission spectrum was recorded in the wavelength region 300-540 nm at $280 \mathrm{~nm}$ excitation. In addition, using the absorption spectrum of propanoic acid, and fluorescence spectrum of BSA, (Concentration ratio of drug and protein is $1: 1$ at $\mathrm{pH}$ 7.4) the fluorescence resonance energy transfer and energy transfer efficacy of BSA with various concentration of drug also evaluated.

Maestro is the graphical user interface for all of Schrodinger's products like CombiGlide ${ }^{\mathrm{TM}}$, Epik ${ }^{\mathrm{TM}}$, Glide $^{\mathrm{TM}}$, Impact ${ }^{\mathrm{TM}}$, Liaison ${ }^{\mathrm{TM}}$, Compoundprep ${ }^{\mathrm{TM}}$, MacroModel $^{\mathrm{TM}}$, Phase ${ }^{\mathrm{TM}}$, Prime ${ }^{\mathrm{TM}}$, QikProp ${ }^{\mathrm{TM}}$, Qsite ${ }^{\mathrm{TM}}$, and Strike ${ }^{\mathrm{TM}}$. It contains tools for building, displaying, and manipulating chemical structures for organizing, loading and storing these structures and associated data, and for setting up, monitoring, and visualizing the results of calculations on these structures.

\section{Result and Discussion}

\section{Absorption characteristics of BSA-PA}

UV-Visible absorption spectroscopy is one of the known simple methods to obtain structural changes of protein and identify the complex formation of the different small molecules [16]. Figure 2(a) shows the absorption spectra of BSA in the presence of PA at concentration ranging from 0-60 $\mu \mathrm{M}$. Both BSA and PA solutions were prepared in Tris-HCL buffer. From the Fig. 2(a) it is observed that the BSA has maximum absorption at $280 \mathrm{~nm}$. The inset in Fig. 2(a) shows absorption spectrum of full view of BSA and its complex of PA. The UV absorption peak of BSA at 280 $\mathrm{nm}$ increases with increase of PA concentrations and the change associated with absorption maximum of BSA Peaks resulting from $n$ - $\mathrm{p}^{*}$ transitions are shifted to shorter wavelengths (blue shift) may be due to the formation of ground state complex between the protein and PA [11]. On increasing the concentration, the band at $330 \mathrm{~nm}$ gets increased in the UV-Vis spectra of BSA and BSA-PA complex. This may be due to the nature of drug (propanoic acid) which has 1-phenylsulfonyl2-methylindole compound in its structure (Fig. 2). The binding constants of complex formation were computed from the change in the intensity to the absorption peak at $280 \mathrm{~nm}$, according to BenesiHildebrand relation shown in Fig. 2(b) and the value of the binding constant $(\mathrm{K})$ for BSA-PA complex is found to be $2.58 \times 10^{4} \mathrm{M}^{-1}$.

\section{Fluorescence quenching mechanism}

In this case, up to a ligand (drug)/protein molar ratio $(d / p)$ of about 1.0 the main effect was the quenching of the protein fluorescence, as evidenced by the spectra in Fig. 3(a). At larger ligand concentration, a new fluorescence emission was initially observed at 382 $\mathrm{nm}$ as a shoulder peak and a red shifted towards 388 $\mathrm{nm}$ as a band was noticed continuously for the the maximum position of the free ligand. This may be due to the hydrophobic environment-around the included species provided by the cyclodextrin [23] cavity which explains this hypsochromic shift. The mechanism and binding constant could be obtained according to the stern volmer plot shown Fig. 3(b). using the classical

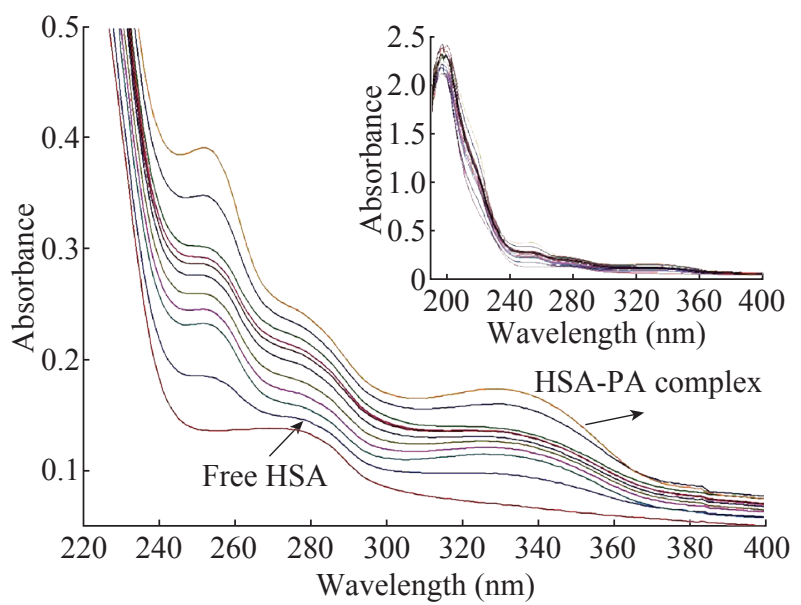

(a)

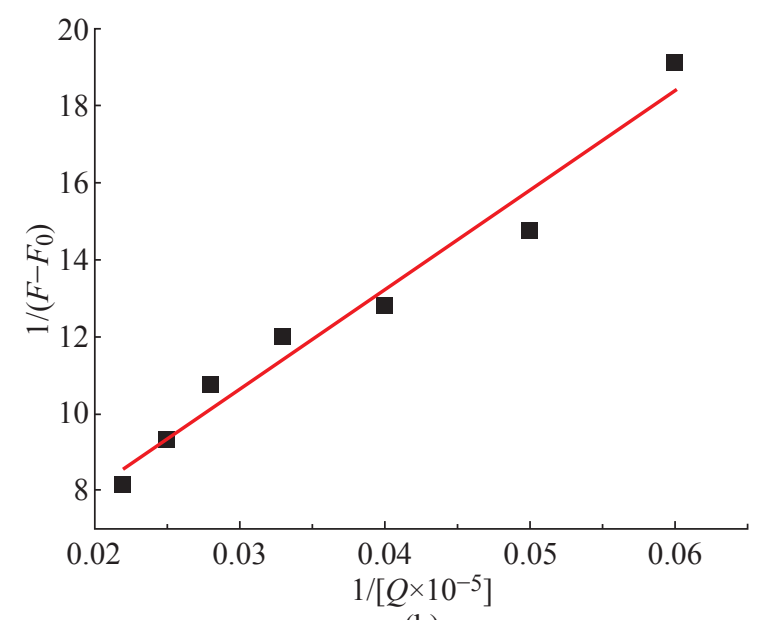

(b)

Fig. 2 (a) The UV absorption spectra of BSA varying with the different concentration of thicarbazonsemi derivative in the distilled water solution at room temperature. Concentrations: BSA $\left(0.1 \times 10^{-5} \mathrm{M}\right)$, propanic acid $(\mathrm{PA})(0.5-7.5 \mu \mathrm{M})$. (b) Benesi-Hildebrand plot of $\operatorname{BSA}\left(0.1 \times 10^{-5} \mathrm{M}\right)$ in the presence of PA. 


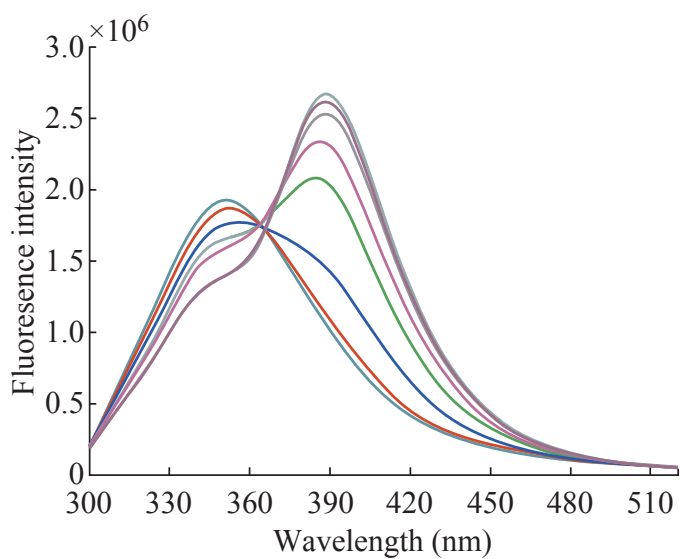

(a)

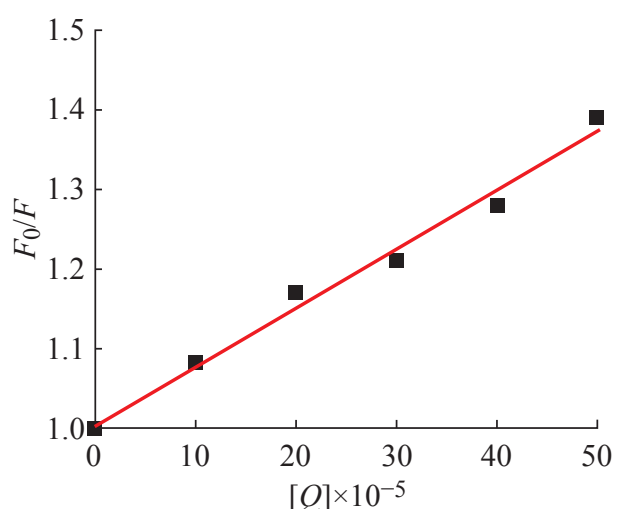

(b)

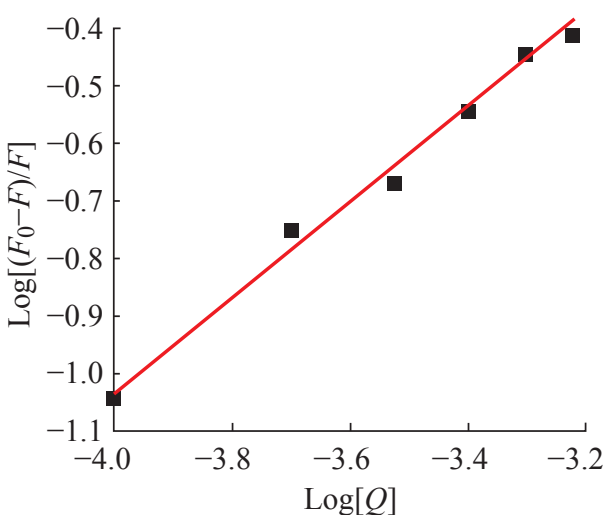

(c)

Fig. 3 (a) Fluorescence emission spectra of BSA-PA $(\mathrm{pH}=7.4)$. BSA $\left(0.1 \times 10^{-5} \mathrm{M}\right)$ in the presence of $(0$ to $7.5 \mu \mathrm{m})$ respectively. (b) Stern volmer plot. (c) Double log plot.

Stern-Volmer (SV) Equation (1)

$F_{0} / F=1+K_{\mathrm{SV}}[Q]$

The $K_{\mathrm{SV}}$ value obtained from the above equation $K_{\mathrm{SV}}=7.72 \times 10^{2} \mathrm{Lmol}^{-1}$. Considering the Stern-Volmer constant of about $10^{2} \mathrm{M}^{-1}$ and the lifetime of albumins of $10^{-8} \mathrm{~s}$ we obtain a value of $10^{10} \mathrm{M}^{-1} \mathrm{~s}^{-1}$ for the rate constant of the bimolecular quenching process [17], which is same to the accepted limit of the rate constant of the diffusional quenching implying biopolymers, $2 \times 10^{10} \mathrm{M}^{-1} \mathrm{~s}^{-1}$. This observation supports the fact that the experimental quenching of albumin fluorescence is may be due to static process.

\section{Energy transfer from BSA to PA}

As there is a decrease in the emission of BSA-PA complex with that of BSA, it was also aimed to study whether there exist Forster's Non radiative energy transfer due to the interaction of BSA with PA or not. According to the Forster dipole-dipole non-radiative energy transfer theory energy transfer is to happen the following conditions $[18,19]$. (1) The donor (emit fluorescence) should have more fluorescence quantum yield, (2) Overlap ( $>30 \%$ ) of the fluorescence emission spectrum of the donor with the absorption spectrum of the acceptor. (3) The distance between the donor and acceptor is within $8 \mathrm{~nm}$.

Energy transfer efficiency $E$ is defined by the following equation [11].

$E=1-\left(F / F_{0}\right)$

where $F$ is the fluorescence intensity of the donor in the presence of acceptor and $F_{0}$ is the fluorescence intensity of the donor. The fluorescence intensity of $\mathrm{BSA}$ is reduced by the energy transfer to PA.

The energy transfer efficiency is related to distance between the acceptor and donor distance and critical energy transfer distance $\left(R_{0}\right)$. The critical energy transfer distance $R_{0}$ and the distance between the acceptor - donor were evaluated using the equation [11] to evaluate the distance between $\operatorname{Trp}$ and propanoic acid.

$E=\frac{R_{0}^{6}}{\left(R_{0}^{6}+r^{6}\right)}$ 
where $R_{0}$ is the critical distance when the transfer efficiency is $50 \%$ and $r$ is the binding distance between the donor and acceptor.

$R_{0}=8.79 \times 10^{-25}\left[k^{2} \mathrm{~N}^{-4} \Phi_{D}\right]$

where $k^{2}$ is the spatial orientation factor of the dipole. $N$ is the average refractive index of the water, $\Phi_{D}$ is the quantum yield of the donor in the absence of the acceptor. $J$ is the overlap integral of the fluorescence emission spectrum of the donor and the absorption spectrum of the acceptor in units $\mathrm{M}^{-1} \mathrm{~cm}^{3} . J$ is given by following equation [20]

$J(\lambda)=\int_{0}^{\infty} F_{D}(\lambda) \varepsilon_{A}(\lambda) \lambda^{4} \mathrm{~d} \lambda$

where $F_{D}(\lambda)$ is the fluorescence intensity of the donor at wavelength $\lambda$ to $\lambda+\Delta \lambda$, with the total intensity normalized to unity and $\varepsilon_{A}(\lambda)$, the molar extinction coefficient of the acceptor at wavelength $(\lambda)$. Figure
4 shows considerable overlap to the fluorescence emission spectrum of BSA with absorption spectrum of

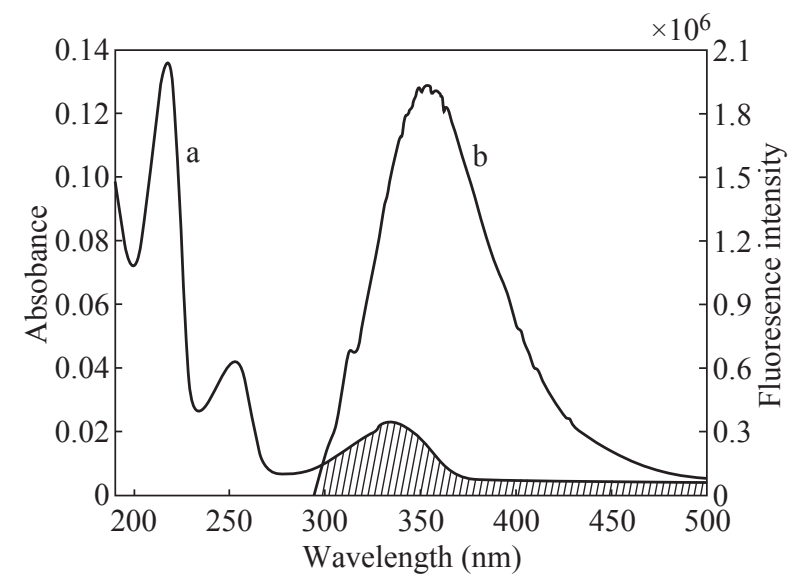

Fig. 4 The overlap of the absorption coefficient spectrum of PA (a) with the fluorescence emission spectrum of BSA (b). ([BSA]: $[\mathrm{PA}]=1: 1), \mathrm{pH}=7.4$.

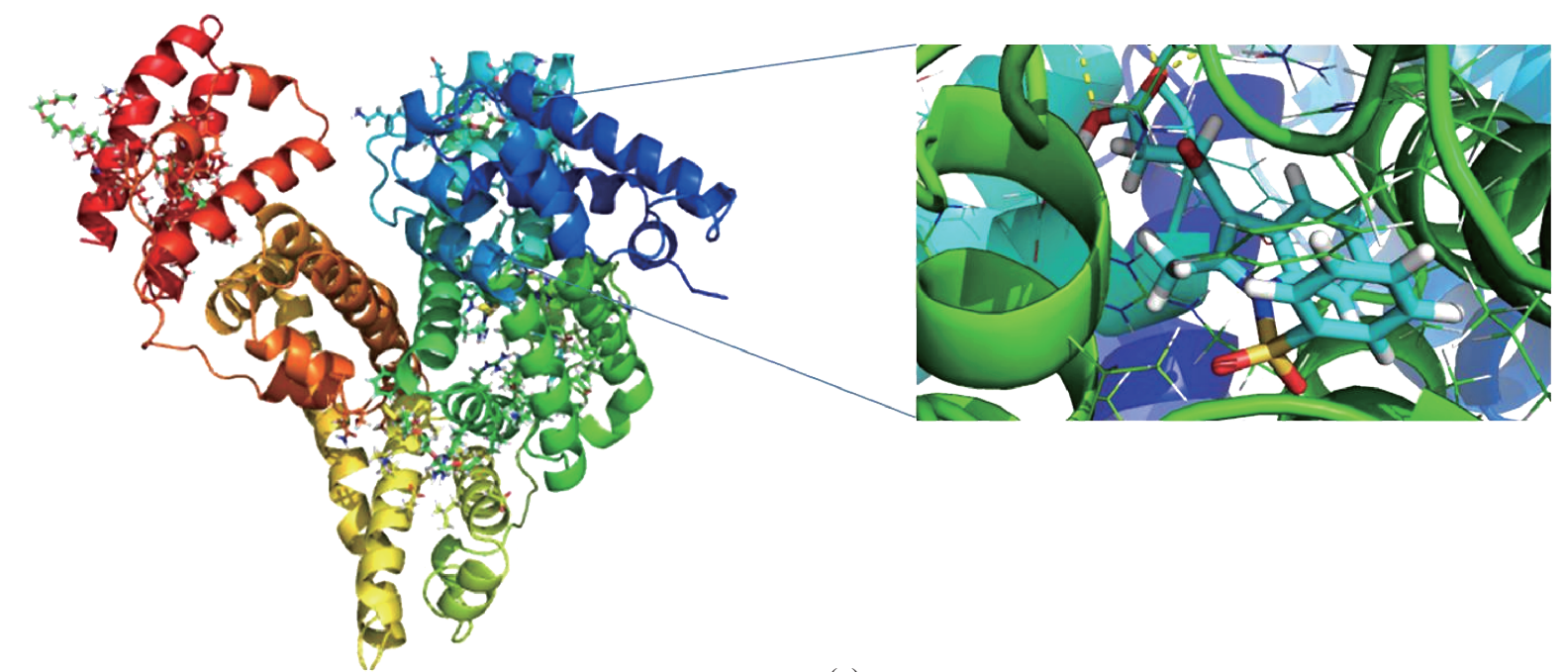

(a)

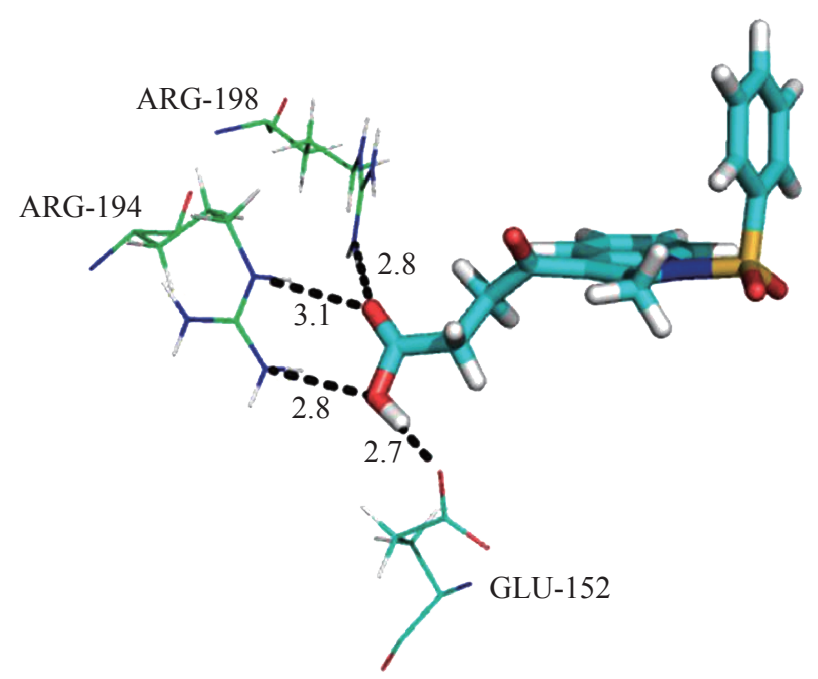

(b)

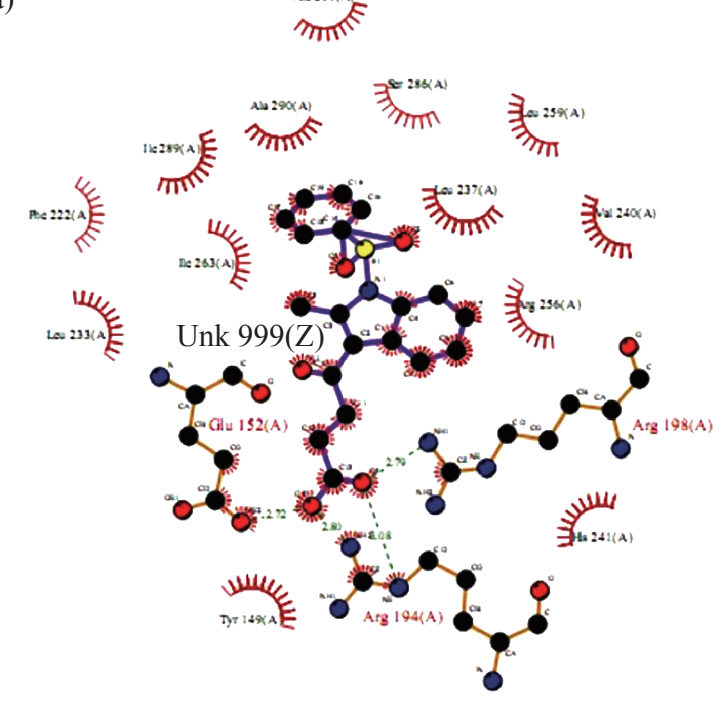

(c)

Fig. 5 (a) Crystal structure of BSA and inset shows BSA-PA complex. (b) pymol view of PA with interacting residues. (c) Hydrogen bonding and hydrophobic interaction of 2D ligplot of BSA-PA complex. 
PA. From the overlapping of the absorption spectrum of the acceptor and fluorescence spectrum of the donor we can calculate the $J$ value. That is $J=3.4482 \times 10^{-21}$ $\mathrm{M}^{-1} \mathrm{~cm}^{3}$. Under the experimental conditions we found $R_{0}=0.466 \mathrm{~nm}$ from equation (3). Using following values $E=0.966, k^{2}=2 / 3, N=1.336$ and $\Phi_{D}=0.118$, $r=1.26 \mathrm{~nm}$. Both $R_{0}$ and $r$ value is lower than the maximum academic values for $R_{0}(5-10 \mathrm{~nm})$ and the donor and acceptor distance $r<8 \mathrm{~nm}$. According to the Forster's non-radiative energy transfer theory indicating the static quenching interaction between PA and BSA. We found both $R_{0}$ and $r$ are in the academic range. These results indicate that the energy transfer from BSA to PA occurs with high probability.

\section{Modeling studies}

Computational binding studies investigate the binding confirmation of BSA and PA. Experimental observation were followed up with molecular docking studies, in which PA was docked to the Bovine serum albumin (BSA) to determine the preferred binding site on the BSA structure. Here two dimensional structure of PA were modeled by using ACD chemsketch software and it was energy minimized using two algorithms, steepest descent and conjugate gradient. The X-ray crystal structure of bovine serum albumin was acquired from protein data bank [24, 25] (PDB ID 4JK4) and it has been further subjected for docking studies using GLIDE Schrödinger suite USA 2009. Protein structure is energy minimized using protein preparation wizard panel to add hydrogen, the charge state of protein residues is important for result generation by Glide. The docking results were good agreement with the experimental results and revealed important pathway between piprazine and BSA. In fluorescence quenching experiments the binding constant of PA with BSA $\mathrm{K}_{\mathrm{b}}$, was evaluated to $7.7 \times 10^{2}$ $\mathrm{L} \mathrm{mol}{ }^{-1}$. According to the formula: $\Delta \mathrm{G}=-\mathrm{RT}\left(\ln \mathrm{K}_{\mathrm{b}}\right)$, as for $\mathrm{T}=298 \mathrm{~K}$, the Gibbs free energy, $\Delta \mathrm{G}$, was figured out to be $-11.5 \mathrm{kcal} \mathrm{mol}^{-1}$. The binding energy $(-10.31$ kcal mol ${ }^{-1}$ ) which was obtained by molecular docking simulation, could be assumed as the Gibbs free energy, $\Delta \mathrm{G}$, approximately. It could be seen that these two values of Gibbs free energies were very close with each other, showing that result obtain from the theoretical analysis were consistent with that using the experimental methods. Docking results showed that PA has good binding affinity in BSA (Fig. 5(a)) (PDB ID 4JK4), also good score and energy for the binding site of BSA and those hydrogen bond and hydrophobic interaction were shown in Fig. 5(b) and (c).

\section{Conclusion}

In summary, UV-vis spectral characterization of BSA, it is observed there is an enhancement of absorption of BSA in presence of PA. This elevated absorption may be attributed to formation of ground state complex due to intermolecular interaction. The observed fluorescence of BSA decreases in the presence of PA is due to the static quenching process. The experimental and modeling studies suggest that PA could bind BSA through the hydrophobic force, and hydrogen bonding. This study reveals that optical spectroscopy techniques could be effectively used to study the design of new drug and understanding their pharmacokinetics.

\section{Acknowledgments}

This study was supported by the Board of Research in Nuclear Sciences, Department of Atomic Energy, Government of India, Project no.2009/34/38/BRNS/3206.

\section{References}

[1] N.C. Howarth, E. Saltzman, S.B. Roberts. Dietary fiber and weight regulation. Nutr. Rev., 2001, 59: 129-139.

[2] E. Fernandez-Garcia, J.U. McGregor. Determination of organic acids during the Fermentation and cold storage of yogurt. J. Dairy Sci., 1994, 77: 2934-2939.

[3] C.L. Bos, D.J. Richel, T. Ritsema, et al., Prostanoids and prostanoid receptors in signal transduction. Int. J. Biochem. Cell Biol., 2004, 36: 1187-1205.

[4] J.M. Wong, R. de Souza, C.W. Kendall, A. Emam, D.J. Jenkins. Colonic health: fermentation and short chain fatty acids. J. Clin. Gastroenterol, 2006, 40: 235-243.

[5] J.R. Lakowicz. Principles of Fluorescence Spectroscopy. Plenum Press, New York, NY, USA, 1983: 44.

[6] P.A. Permana, C. Menge, P.D. Reaven. Macrophagesecreted factors induceadipocyte inflammation and insulin resistance. Biochem. Biophys. Res. Commun., 2006, 341: 507-514.

[7] G. Boden. Obesity and free fatty acids, Endocrinol. Metab. Clin. North Am., 2008, 37: 635-646.

[8] A. Kennedy, K. Martinez, C.C. Chuang, K. La Point, M. McIntosh. Saturated fatty acid-mediated inflammation and insulin resistance in adipose tissue: mechanisms of action and implications. J. Nutr., 2009, 139: 1-4.

[9] R. Micha, D. Mozaffarian. Trans fatty acids: effects on metabolic syndrome, heart disease and diabetes. Nat. Rev. Endocrinol, 2009, 5: 335-344.

[10] A.M. Sharma, V.T. Chetty. Obesity, hypertension and insulin resistance. Acta Diabetol. 2005, 42: S3-S8.

[11] S. Chinnathambi, D. Velmurugan, N. Hanagata, P. Aruna, S. Ganesan. Investigations on the interactions of 5-fluorouracil with bovine serum albumin: Optical spectroscopic and molecular modeling studies. J. Lumin., 2014, 151: 1-10. 
[12] C.N. Yan, H.X. Zhang, P. Mei, Y. Liu. Study on binding reaction between flucytosine and bovine serum albumin. Chin. J. Chem., 2005, 23: 1151-1156.

[13] R.T. Liu, P.F. Qin, L. Wang, et al., Toxic Effects of ethanol on bovine serum albumin. J. Biochem. Mol. Toxicol, 2010, 24: 66-71.

[14] P. Bourassa, C.D. Kanakis, P. Tarantilis, et al., Resveratrol, genistein, and curcumin bind bovine serum albumin. $J$. Phys. Chem. B, 2010, 114: 3348-3354.

[15] S. Dubeau, P. Bourassa, T.J. Thomas, H.A. TajmirRiahi. Biogenic and synthetic polyamines bind bovine serumalbumin. Biomacromolecules, 2010, 11: 1507-1515.

[16] Y. Qian, X. Zhou, J. Chen, Y. Zhang. Binding of bezafibrate to human serum albumin: insight into the noncovalent interaction of an emerging contaminant with biomacromolecules. Molecules, 2012, 17: 6821-6831.

[17] Y.Q. Wang, H.M. Zhang, G.C. Zhang, W.H. Tao, S.H. Tang. Binding of brucine to human serum albumin. $J$. Mol. Struct., 2007, 830: 40-45.

[18] B.K. Paul, N. Guchhait. Modulation of Prototropic Activity and Rotational Relaxation Dynamics of a Cationic Biological Photosensitizer within the Motionally Constrained Bio-environment of a Protein. J. Phys. Chem. B, 2011, 115: 10322-10334.

[19] A.M. Rossi, C.W. Taylor. Analysis of protein-ligand interactions by fluorescence polarization. Nat. Protoc., 2001, 6: 365-387.

[20] L. Stryer, R.P. Haugland. Energy transfer: a spectroscopic ruler. Proc. Natl. Acad. Sci., 1967, 58: 719-726.
[21] R. Krishna, P.G. Aravindan, M. Yogavel, et al., 1,2,3,4,4a, 12a-Hexahydro-2, 5, 5-trimethyl- $1 \mathrm{H}$-[2] benzopyrano[3,2-c]coumarin. Acta Cryst., 2003, E59: o875-0877.

[22] E.M. Martin Del Valle. Cyclodextrins and their uses: a review. Process Biochem, 2003, 39: 1033-1046.

[23] V. Oana, V. Tintaru, V. Gavriliu, V. Maior, M.J. Hillebrand. Spectral Study and Molecular Modeling of the Inclusion Complexes of $\beta$-Cyclodextrin with Some Phenoxathiin Derivatives. Phys. Chem., 2002, 106: 257263.

[24] B. Sekula, K. Zielinski, A. Bujacz. Crystallographic studies of the complexes of bovine and equine serum albumin with 3,5-diiodosalicylic acid. Int. J. boil. Macromol, 2013, 60: 316-324.

[25] A.J. Ryan, C.W. Chung, S. Curry. Crystallographic analysis reveals the structural basis of the high-affinity binding of iophenoxic acid to human serum albumin. BMC Struct. Biol., 2011, 18: 11-18.

Copyright $(2015$ Subramani Karthikeyan, Shanmugavel Chinnathambi, Devadasan Velmurugan, Ganesan Bharanidharan and Singaravelu Ganesan. This is an open-access article distributed under the terms of the Creative Commons Attribution License, which permits unrestricted use, distribution, and reproduction in any medium, provided the original author and source are credited. 